\title{
The New American Joint Committee on Cancer T Staging System for Stomach: Increased Complexity without Clear Improvement in Predictive Accuracy for Endoscopic Ultrasound
}

Chaoqun Han(Former Corresponding Author)

Wuhan Union Hospital https://orcid.org/0000-0003-1338-865X

Xu Tao

Wuhan Union Hospital

Qin Zhang

Wuhan Union Hospital

Jun Liu

Wuhan Union Hospital

Zhen Ding(New Corresponding Author) ( $\square$ docd720@126.com )

Xiaohua Hou

Wuhan Union Hospital

Research article

Keywords: Endoscopic Ultrasound; gastric cancer; American Joint Committee on Cancer; staging; accuracy

Posted Date: February 21st, 2020

DOI: https://doi.org/10.21203/rs.2.24210/v1

License: (a) This work is licensed under a Creative Commons Attribution 4.0 International License. Read Full License 


\section{Abstract}

Background: The efficacy of endoscopic ultrasound (EUS) for determining T category is variable for gastric cancer. The aim of this study is to evaluate the superiority of EUS by using the $6^{\text {th }}$ edition American Joint Committee on Cancer (AJCC) staging system for stomach cancer compared to the new $7^{\text {th }} / 8^{\text {th }}$ edition.

Methods: A retrospective analysis of clinical and EUS imaging features of 348 gastric carcinoma patients who underwent radical resection were collected. Differences between the $6^{\text {th }}$ edition and $7^{\text {th }} / 8^{\text {th }}$ edition $T$ staging system for EUS preoperative evaluated were compared.

Results: The accuracy of EUS T staging was $72.4 \%$ for $7^{\text {th }} / 8^{\text {th }}$ edition and $78.4 \%$ for $6^{\text {th }}$ edition; especially the T3 stage accuracy was significantly worse when T3 group status was changed. The tumor location, EUS type and histological type were associated with inaccuracy. We further analyzed the EUS image features for each tumor T stage and found muscularis propria (MP) visible indistinctly or obvious thickening were considered to be indicators that lesions involved to the MP with a sensitivity of $81.3 \%$; MP disappeared completely and accompanied with serosal layer intact might be a marker that lesion invaded to the subserosa; We also found that irregularities in the outer edge of the gastric wall were markers of gastric serosal layer penetration with a positive predictive value of $92.2 \%$.

Conclusions: The increased complexity of the $7^{\text {th }} / 8^{\text {th }}$ edition $T$ staging system is accompanied by worsening in the predictive accuracy for EUS as compared to the $6^{\text {th }}$ edition. Furthermore, the tumor location, EUS type, histological type and EUS image features for each tumor T stage should warrant attention.

\section{Background}

Gastric cancer is the fourth most common malignancy and the second leading cause of cancer-related death worldwide[1]. It also has a poor prognosis and high mortality[2, 3]. Accurate categorization of the tumor stage, including the invasive depth and lymph node status, is crucial for prognostic assessment and making initial therapeutic decisions for patients.

Currently, the tumor-lymph node-metastasis (TNM) staging system based on the anatomic extent of malignancies has been a benchmark for prognosis evaluation. In order to maintain the staging system relevant, the American Joint Committee on Cancer (AJCC) and the International Union Against Cancer have collaborated on aperiodic revisions of this staging system, leading to the 6th edition in 2002, the 7th edition in 2010[4], and the 8th edition in 2017[5, 6].

For gastric cancer, several changes compared to the 6 th edition were made. This new edition, the $T$ classification categories have been redefined. According to the 7th /8th edition, the 6th edition T2b classification was redefined into T3 (subserosa) and T3 stage was classified into T4a (serosa). With this 
complicated revisions, there are improvements for prognostic value in terms of homogeneity, discriminatory ability, and monotonicity of gradients for patients with gastric cancer[3, 7].

However, accurate preoperative staging is important for selecting the most effective treatment. Endoscopic ultrasonography (EUS) provides ability to differentiate anatomic structural layers of the gastric wall and show remarkable differences in their echogenic appearance if tumor invaded[8]. EUS is indeed considered to be the best-choice imaging modality for local and regional staging of gastric cancer compared to other methods, especially for determining T category[9-12]. Although the 7th /8th edition TNM staging system provide a more detailed classification, they seem to be more complex to apply than the 6th edition. In our study, we aimed to evaluate the validity of EUS compared the 6th edition to the 7th /8th edition of the AJCC T staging system for gastric cancer; Furthermore, we attempted to identify sonographic features that affect the accuracy of EUS staging.

\section{Methods}

\section{Patient Selection}

A total of 1044 patients with gastric cancer performed at our center from December 2015 to December 2018 were included. To obtain correct histological staging, all patients included in this analysis met the following criteria: (1) their disease was pathologically defined as gastric cancer; (2) they underwent tumor-free resection (R0) margin status; (3) they underwent pre-operative stage by using EUS. The exclusion criteria were (1) surgery that was preceded by preoperative chemoradiotherapy (neoadjuvant therapy) or palliative surgery; (2) patients with distant metastasis; (3) patients with synchronous malignancies or previous other primary cancers; (4); tumors with bulky obstruction that EUS failed to pass through. The study was approved by the Ethics Committee of Tongji Medical College, Huazhong University of Science and Technology (No: IORG0003571). All patients signed informed consent for EUS operation and data had been anonymized and de-identified.

\section{EUS equipment and technique procedures}

Curved heteroscope with a 360-degree radial echoendoscope (Olympus processor EU-ME2ðOlympus, Tokyo, Japan) were used. $300 \mathrm{ml}$ to $800 \mathrm{ml}$ of de-aerated water or fitted with a water-filled balloon to assist acoustic coupling and to improve transmission of the ultrasound beam with variable frequencies of $7.5,10$, and $12 \mathrm{~Hz}$. Analogous to pathologic classification, the extent of wall invasion was imaged as a hypoechoic disruption and evaluated based on the tumor infiltration into each layer [13]. Assessment of tumor invasion depth by EUS and all patients were restaged using the $6^{\text {th }}, 7^{\text {th }}$ and $8^{\text {th }}$ edition of the AJCC TNM staging systems $[14,15]$. The three different T stage versions were showed in Table1. All operations were performed by an experienced gastroenterologist with a track record of more than 1000 EUS per year.

\section{Data collection}


In order to compare the difference of EUS efficacy between AJCC6 ${ }^{\text {th }}$ edition and AJCC $7^{\text {th }} / 8^{\text {th }}$ edition, the EUS accuracy was determined by comparing tumor depth on EUS to tumor depth on pathology. We also focused on the factors that may be influenced accurate diagnosis of EUS tumor invasion depth. Data included patient demographics, clinicopathologic details and ultrasonic characteristics. Challenge in the identification of nodes with EUS is the inability to visualize nodes that are outside the range of the transducer [16-18]. Thus, gastric cancer $\mathrm{N}$ staging remains to be an area of uncertainty. The efficacy of EUS $\mathrm{N}$ staging and other related data are not shown.

\section{Statistical analysis}

All patients were restaged using the $6^{\text {th }}$ and $7^{\text {th }} / 8^{\text {th }}$ editions of the AJCC T staging system. For statistical analysis, categorical variable results are presented as numbers and percentages, and continuous variables are presented as mean \pm standard deviation (SD). The possible influence of variously categorical or non-categorical variance were conducted by Pearson's chi-squared test and t tests. Subsequently, logistic regression models were performed to assess potential associations relate to EUS accuracy. Statistical analysis was performed using IBM SPSS Statistics software (version 20.0, IBM Corp, Armonk, NY, USA). A significance level of $\mathrm{P} \leq 0.05$ was used for all models. (two-sided).

\section{Results}

\section{General Patient Characteristics}

In total, 348 patients were included in this study. The patients ranged in age from 29 to 77 years with a mean age of 56.7 years, and $61.2 \%$ were male. With consideration to the location of lesions, the main tumor occurrence was located in the antrum and corpus (64.4\%); nearly two-third of cancer in this group and T3 stage accounted for the majority of cases. Tumors were well differentiated in $11.2 \%$, moderately differentiated in $13.8 \%$, poorly differentiated in $47.4 \%$ and signet ring cell adenocarcinoma in $27.6 \%$, respectively. We also identified EUS image characteristics, including presence of circumferential lesions (cancer extension beyond a semi-circular area, $34.5 \%$ of tumors were circumferential lesions $\geq 1 / 2$ ), and ascites (8.6\%). The clinicopathological characteristics are summarized in Table 2.

\section{Surgical and pathological results}

Table 2 shows the detailed classifications based on the $6^{\text {th }}$ and $7^{\text {th }} / 8^{\text {th }}$ editions of the TNM classification. Among the major revisions in the $7^{\text {th }} / 8^{\text {th }}$ edition T classification was that the definition of T3 and T4 stage was changed. According to the $6^{\text {th }}$ edition T staging, T1, T2, T3 and T4 were seen in $45(13.0 \%)$, 123 (35.3\%), 174 (50.0\%) and 6 (1.7\%) respectively. However, T2, T3 and T4 were seen in 24 (6.9\%), 99 $(28.4 \%)$, and $180(51.7 \%)$, respectively depend on $7^{\text {th }} / 8^{\text {th }}$ edition staging systems. Among 348 patients, the most change was assigned a more T4 stage numbers and less T2 and T3 classification according to the AJCC $7^{\text {th }} / 8^{\text {th }}$ edition. The redefined $T 2, T 3$, and T4 classifications might be more evenly distributed.

\section{Efficacy of EUS in Classifying T stage}


Compared with pT category, the overall accuracy of EUS for T staging were $72.4 \%$ on $7^{\text {th }} / 8^{\text {th }}$ edition staging systems respectively; and $78.4 \%$ on $6^{\text {th }}$ edition staging system respectively; Detailed comparisons between UT and pT categories are presented in Table 3. Furthermore, the differences in accuracy for the four stages were statistically significant $\left(P=0.001\right.$ for $7^{\text {th }} / 8^{\text {th }}$ edition and $P=0.001$ for $6^{\text {th }}$ edition, respectively). On the whole, the frequency of over-stage by EUS nearly be equal to under-stage ( $14.7 \%$ vs. $12.9 \%)$

For AJCC $7^{\text {th }} / 8^{\text {th }}$ edition, EUS had the highest accuracy in pT4 patients. However, note that as many as $36.4 \%$ of pT3 patients were overstaged as having uT4 lesions by EUS. In pT2 cases, 50.0\% were accurately classified, but as many as $25.5 \%$ were understaged as uT1 lesions by EUS. For AJCC $6^{\text {th }}$ edition, EUS also had the highest accuracy in pT4 patients. Unexpectedly, the accuracy of EUS in classifying the T3 category was up to $89.7 \%$, and $10.3 \%$ of uT2 patients identified by EUS were understaged from pT3 cases. It is also interesting found that nearly two-thirds (61.0\%) of pT2 patients were accurate diagnosis. The accuracy of EUS for T2 and T3 stage were obviously improved based on AJCC $6^{\text {th }}$ edition.

With regard to T1 cases, our data showed that EUS had a relatively satisfactory accurate rate. However, it should also be noted that $20 \%$ of pT1 patients were actually could be assessed by endoscopic minimally invasive resection.

\section{Factors influencing EUS gastric cancer staging}

Among the patients included in the study, EUS accuracy was not influenced by the cancer diameter or presence of ascites or not. Interestingly, as shown in table 4, EUS had the highest accuracy for corpus $(86.7 \%)$, and tended to decline in lesions located in the upper third of the stomach. Multivariate logistic regression analysis showed that corpus, radial scanning and well-differentiated were associated with higher accuracy of EUS(p『0.05). The gastric angle seems to present a significant overstaging $(p=0.001)$. The fundus and gastroesophageal junction had a greater possibility of understaging $(p=0.014)$. Further multivariate logistic regression analysis indicated the lesions located in gastric angle presented a significantly higher risk of overstaging ( $p=0.018, O R=2.278$; Table 5$)$. The accuracy of EUS was also influenced by the EUS type. Linear array presented a significant incorrect staging $(p=0.012)$ and had a greater possibility of understaging $(p=0.009)$. There were also significant differences in the accuracy among each histological type $(p=0.039)$. For well-differentiated tumors, EUS had better staging accuracy relative to that for signet ring cell carcinoma (84.6\% vs. 62.5\%). Furthermore, when subjected to multivariate analysis, lesions with signet ring cell adenocarcinoma presented significant risk factors for inaccuracy with a 2.684-fold Odds Ratio $(p=0.001)$ and understaging with 4.800-fold-Odds Ratio( $(p=0.005)$.

\section{The EUS image features for different tumor $T$ stages}


We then reread all patients' results, analyzed EUS image feature and found that the hypoechoic change of the first three layer (the mucosal layer to the submucosal layer) was a feature for T1 (stage)(Figure1A). If accompanied with muscularis propria (MP) visible indistinctly or obvious thickening were considered to be indicators that lesion involved to the MP (T2 stage) (Figure1B-C). The sensitivity between EUS and pathological results for this T2 stage feature was $81.3 \%$ and had a high positive predictive value (PPV) 83.3\%. Furthermore, when MP disappeared completely and accompanied with serosal layer intact was a marker that lesion involved to the subserosa (T3 stage) (Figure1D). The consistency rate between EUS and pathological results was $75.8 \%$. Finally, we also found that irregularities in the outer edge of the gastric wall were markers of gastric serosal layer penetration (Figure1E). The consistency rate between EUS and pathological results for serosal involvement was $82.6 \%$. Sensitivity, specificity, PPV and negative predictive values (NPV) for this characteristic were $84.9 \%, 70.1 \%, 92.2 \%$ and $55.2 \%$, respectively. Gastric wall outer edge irregularity is an effective indicator for confirming serosal extension. The Figure 1 depicted the EUS features of each T stage.

\section{Discussion}

Tumor depth is an important component of guiding the treatment of gastric cancer. The current study describes the impact of the EUS accurate changes for stomach cancer by comparing the 6th and 7th /8th edition of the T staging system. The key findings of this paper are the following: (1). Compared with 7th /8th edition, the 6th edition T staging system might be more accurate and more adaptable for EUS T stage; (2). the tumor location, EUS type and histological type were associated with inaccuracy; (3). The EUS image features of each tumor T stage could guide judgment for EUS gastroenterologist.

The reinforced TNM classification system attempts to determine extent of disease, providing guidance for treatment planning, and predicting outcome[19]. Most authors approve that increased complexity of the 7th /8th edition system is superior to the 6th edition system, especially in evaluating the prognosis of cancer patients [20-22], however, the impact for EUS T stage remains unclear. A most substantial major difference between AJCC6th and AJCC7th /8th is that the T2 stage in the 6th edition system was subclassified into T2 and T3 in the 7th /8th edition system. After revised, the accuracy in classifying the T2 and T3 category are not satisfactory, just for only $50.0 \%$ and $42.4 \%$ in our study, respectively. However, when the T3 uT category was defined as T2 by 6th edition, the results could be clearly distinguished and accuracy could be reached $61.0 \%$ and $89.7 \%$, respectively.

According to the new edition system,the 7th /8th edition strengthens the role of subserosalhowever, from a technical perspective, distinguishing between subserosal and serosal lesions by EUS is challenging. This is the main reason for the poor accuracy of T3 stage. However, we found that MP disappeared completely and accompanied with serosal layer intact might be a marker that lesion involved to the subserosa. The consistency rate was $75.8 \%$. For all this, the challenge of accurate T3 stage still remains a frequent issue since this $T$ stage tend to be overstaging. In some certain area, the lesser curvature regions and fundal, the gastric wall is not entirely covered by the serosa[23], which may result in overstaging. 
Next, we also found that MP visible indistinctly or obvious thickening were markers that lesion involved to the T2 stage and had a high PPV (83.3\%). However, mild thickening of the MP layer may be not only due to cancer infiltration (T2 stage) but also inflammatory reaction (T1 stage). Moreover, fibrosis and edema that produced hypoechoic changes also made EUS evaluation confusion[24, 25]. For T4 stage, according to the AJCC 7th /8th system, tumors involved with serosa could be categorized as T4, most of primary T3 patients by AJCC 6th system were placed in the stage T4. The T4 staging accuracy was the highest in pre-operative staging. This result indicates that EUS is an effective method for evaluating serosal invasion, largely because gastric wall outer edge irregularity is a good indicator of cancer invasion (PPV nearing 93\%).

In addition, the location of tumor, histological and EUS type are associated with accuracy of EUS staging, where in case with tumor located in gastric angle was an independent indicator that was associated with EUS overstaged. The EUS accuracy was higher in well-differentiated histological type than other parts; and tumors in signet ring cell adenocarcinoma were related with EUS understaged. The reason may be that tumors differentiate into signet ring cell adenocarcinoma are commonly scirrhous and tend to have tumor microinvasion that cannot be detected by EUS[26]. These results suggested that cancers with these features may be more severe than that indicated by EUS pre-operative staging. Careful attention is required during EUS examination must precede therapeutic schedule for gastric cancer with these characteristics.

Certainly, the present study has some limitations that require further discussion. First, the EUS staging were performed by using retrospectively still images. Reviewing still images did not provide the information about lesion flexibility to predict invasion depth. Secondly, the sample of patients is relatively small and limits the application of the results; third, $T$ stage with including a subgroup, such as T1a vs. T1b, T4a vs. T4b, could be further discussion. Finally, the EUS accuracies for N/M staging are not compared is another limitation that should be considered in this study. A multicenter prospective study is required.

\section{Conclusions}

In conclusion, EUS could serve as an accurate technology to determine the infiltration depth of gastric cancer. In view of the T stage, the 6th T stage edition system might be suited for EUS T stage; Gastric cancers with location, histological and EUS type were more frequently associated with incorrect staging. For these patients, it is recommended that gastroenterologists should considered the $T$ stage image characteristics we mentioned above.

\section{Declarations}

\section{Abbreviations}


AJCC: American Joint Committee on Cancer; EUS: Endoscopic Ultrasound; MP: muscularis propria; TNM: tumor-lymph node-metastasis; SD: standard deviation; PPV: positive predictive value; NPV: negative predictive value

\section{Ethics approval and consent to participate}

The study was approved by the Ethics Committee of Tongji Medical College, Huazhong University of Science and Technology (IORG No: IORG0003571). All patients signed informed consent for EUS operation and data had been anonymized and de-identified.

\section{Consent to publish}

Not applicable.

\section{Availability of data and materials}

The datasets used and/or analyzed during the current study are available from the corresponding author on reasonable request.

\section{Competing interests}

All authors state that they have no conflict of interest.

\section{Funding}

This study was supported in part by the National Natural Science Foundation of China (No. 81800467 , 81720108006, 81330014, 81770637).

\section{Authors' contributions}

$\mathrm{HC}$ was involved in the conception, design and drafted the paper. TX and LJ were involved in the analysis and interpretation of the data. ZQ was analyzed of pathological results. HX checked the research outcomes. DZ revised it critically for intellectual content. All authors gave the final approval of the version to be published.

\section{Acknowledgments}

The authors thank all participants for accomplishing this study.

\section{References}

1. Zhang J, Zhou Y, Jiang K, Shen Z, Ye Y, Wang S: Evaluation of the seventh AJCC TNM staging system for gastric cancer: a meta-analysis of cohort studies. Tumour biology : the journal of the International Society for Oncodevelopmental Biology and Medicine 2014, 35(9):8525-8532. 
2. Sitarz R, Skierucha M, Mielko J, Offerhaus GJA, Maciejewski R, Polkowski WP: Gastric cancer: epidemiology, prevention, classification, and treatment. Cancer management and research 2018, 10:239-248.

3. Ji X, Bu ZD, Yan Y, Li ZY, Wu AW, Zhang LH, Zhang J, Wu XJ, Zong XL, Li SX et al: The 8th edition of the American Joint Committee on Cancer tumor-node-metastasis staging system for gastric cancer is superior to the 7th edition: results from a Chinese mono-institutional study of 1663 patients. 2018, 21(4):643-652.

4. Dikken JL, van de Velde CJ, Gonen M, Verheij M, Brennan MF, Coit DG: The New American Joint Committee on Cancer/International Union Against Cancer staging system for adenocarcinoma of the stomach: increased complexity without clear improvement in predictive accuracy. Annals of surgical oncology 2012, 19(8):2443-2451.

5. He X, Wu W, Lin Z, Ding Y, Si J, Sun LM: Validation of the American Joint Committee on Cancer (AJCC) 8th edition stage system for gastric cancer patients: a population-based analysis. Gastric cancer : official journal of the International Gastric Cancer Association and the Japanese Gastric Cancer Association 2018, 21(3):391-400.

6. Amin MB, Greene FL, Edge SB, Compton CC, Gershenwald JE, Brookland RK, Meyer L, Gress DM, Byrd DR, Winchester DP: The Eighth Edition AJCC Cancer Staging Manual: Continuing to build a bridge from a population-based to a more "personalized" approach to cancer staging. CA: a cancer journal for clinicians 2017, 67(2):93-99.

7. Liu JY, Peng CW, Yang XJ, Huang CQ, Li Y: The prognosis role of AJCC/UICC 8(th) edition staging system in gastric cancer, a retrospective analysis. American journal of translational research 2018, 10(1):292-303.

8. Cardoso R, Coburn N, Seevaratnam R, Sutradhar R, Lourenco LG, Mahar A, Law C, Yong E, Tinmouth $\mathrm{J}$ : A systematic review and meta-analysis of the utility of EUS for preoperative staging for gastric cancer. Gastric cancer : official journal of the International Gastric Cancer Association and the Japanese Gastric Cancer Association 2012, 15 Suppl 1:S19-26.

9. Mehmedovic A, Mesihovic R, Saray A, Vanis N: Gastric cancer staging: EUS and CT. Medical archives (Sarajevo, Bosnia and Herzegovina) 2014, 68(1):34-36.

10. Han C, Lin R, Shi H, Liu J, Qian W, Ding Z, Hou X: The role of endoscopic ultrasound on the preoperative T staging of gastric cancer: A retrospective study. Medicine 2016, 95(36):e4580.

11. Wu CX, Zhu ZH: Diagnosis and evaluation of gastric cancer by positron emission tomography. World journal of gastroenterology 2014, 20(16):4574-4585.

12. Cho JW: The role of endoscopic ultrasonography in T staging: early gastric cancer and esophageal cancer. Clinical endoscopy 2013, 46(3):239-242.

13. Merkow RP, Herrera G, Goldman DA, Gerdes H, Schattner MA, Markowitz AJ, Strong VE, Brennan MF, Coit DG: Endoscopic Ultrasound as a Pretreatment Clinical Staging Tool for Gastric Cancer: Association with Pathology and Outcome. Annals of surgical oncology 2017, 24(12):3658-3666. 
14. Qiu MZ, Wang ZQ, Zhang DS, Liu Q, Luo HY, Zhou ZW, Li YH, Jiang WQ, Xu RH: Comparison of 6th and 7th AJCC TNM staging classification for carcinoma of the stomach in China. Annals of surgical oncology 2011, 18(7):1869-1876.

15. Ye J, Ren Y, Wei Z, Hou X, Dai W, Cai S, Tan M, He Y, Yuan Y: External validation of a modified 8th AJCC TNM system for advanced gastric cancer: Long-term results in southern China. Surgical oncology 2018, 27(2):146-153.

16. Grotenhuis BA, Wijnhoven BP, Poley JW, Hermans JJ, Biermann K, Spaander MC, Bruno MJ, Tilanus $\mathrm{HW}$, van Lanschot JJ: Preoperative assessment of tumor location and station-specific lymph node status in patients with adenocarcinoma of the gastroesophageal junction. World journal of surgery 2013, 37(1):147-155.

17. Seevaratnam R, Cardoso R, McGregor C, Lourenco L, Mahar A, Sutradhar R, Law C, Paszat L, Coburn $\mathrm{N}$ : How useful is preoperative imaging for tumor, node, metastasis (TNM) staging of gastric cancer? A meta-analysis. Gastric cancer : official journal of the International Gastric Cancer Association and the Japanese Gastric Cancer Association 2012, 15 Suppl 1:S3-18.

18. Wang X, Gao Y, Li J, Wu J, Wang B, Ma X, Tian J, Shen M, Wang J: Diagnostic accuracy of endoscopic ultrasound, computed tomography, magnetic resonance imaging, and endorectal ultrasonography for detecting lymph node involvement in patients with rectal cancer: A protocol for an overview of systematic reviews. Medicine 2018, 97(43):e12899.

19. Ahn HS, Lee HJ, Yoo MW, Kim SG, Im JP, Kim SH, Kim WH, Lee KU, Yang HK: Diagnostic accuracy of $\mathrm{T}$ and $\mathrm{N}$ stages with endoscopy, stomach protocol $\mathrm{CT}$, and endoscopic ultrasonography in early gastric cancer. Journal of surgical oncology 2009, 99(1):20-27.

20. Lu J, Zheng CH, Cao LL, Li P, Xie JW, Wang JB, Lin JX, Chen QY, Lin M, Tu RH et al: Comparison of the 7th and 8th editions of the American joint committee on cancer TNM classification for patients with stage III gastric cancer. Oncotarget 2017, 8(48):83555-83562.

21. Lu J, Zheng ZF, Xie JW, Wang JB, Lin JX, Chen QY, Cao LL, Lin M, Tu RH, Huang CM et al: Is the 8th Edition of the AJCC TNM Staging System Sufficiently Reasonable for All Patients with Noncardia Gastric Cancer? A 12,549-Patient International Database Study. Annals of surgical oncology 2018, 25(7):2002-2011.

22. Chen QY, Zheng CH, Li P, Xie JW, Wang JB, Lin JX, Lu J, Cao LL, Lin M, Tu RH et al: A long-term conditional survival analysis for gastric cancer based on 7th and 8th TNM classification in Eastern and Western populations. European journal of surgical oncology : the journal of the European Society of Surgical Oncology and the British Association of Surgical Oncology 2018, 44(12):19491954.

23. Bando E, Kawamura T, Kinoshita K, Takahashi S, Maeda A, Osada S, Tsubosa Y, Yamaguchi S, Uesaka K, Yonemura Y: Magnitude of serosal changes predicts peritoneal recurrence of gastric cancer. Journal of the American College of Surgeons 2003, 197(2):212-222.

24. Bentrem D, Gerdes H, Tang L, Brennan M, Coit D: Clinical correlation of endoscopic ultrasonography with pathologic stage and outcome in patients undergoing curative resection for gastric cancer. 
Annals of surgical oncology 2007, 14(6):1853-1859.

25. Han C, Lin R, Zhang Q, Liu J, Ding Z, Hou X: Role of endoscopic ultrasound-guided fine needle aspiration in the diagnosis of mass lesions. Experimental and therapeutic medicine 2016, 12(2):1085-1092.

26. Yokota T, Kunii Y, Teshima S, Yamada Y, Saito T, Kikuchi S, Yamauchi H: Signet ring cell carcinoma of the stomach: a clinicopathological comparison with the other histological types. The Tohoku journal of experimental medicine 1998, 186(2):121-130.

\section{Tables}

Due to technical limitations, Tables 1 - 5 are only available for download from the Supplementary Files section.

\section{Figures}


Figure1

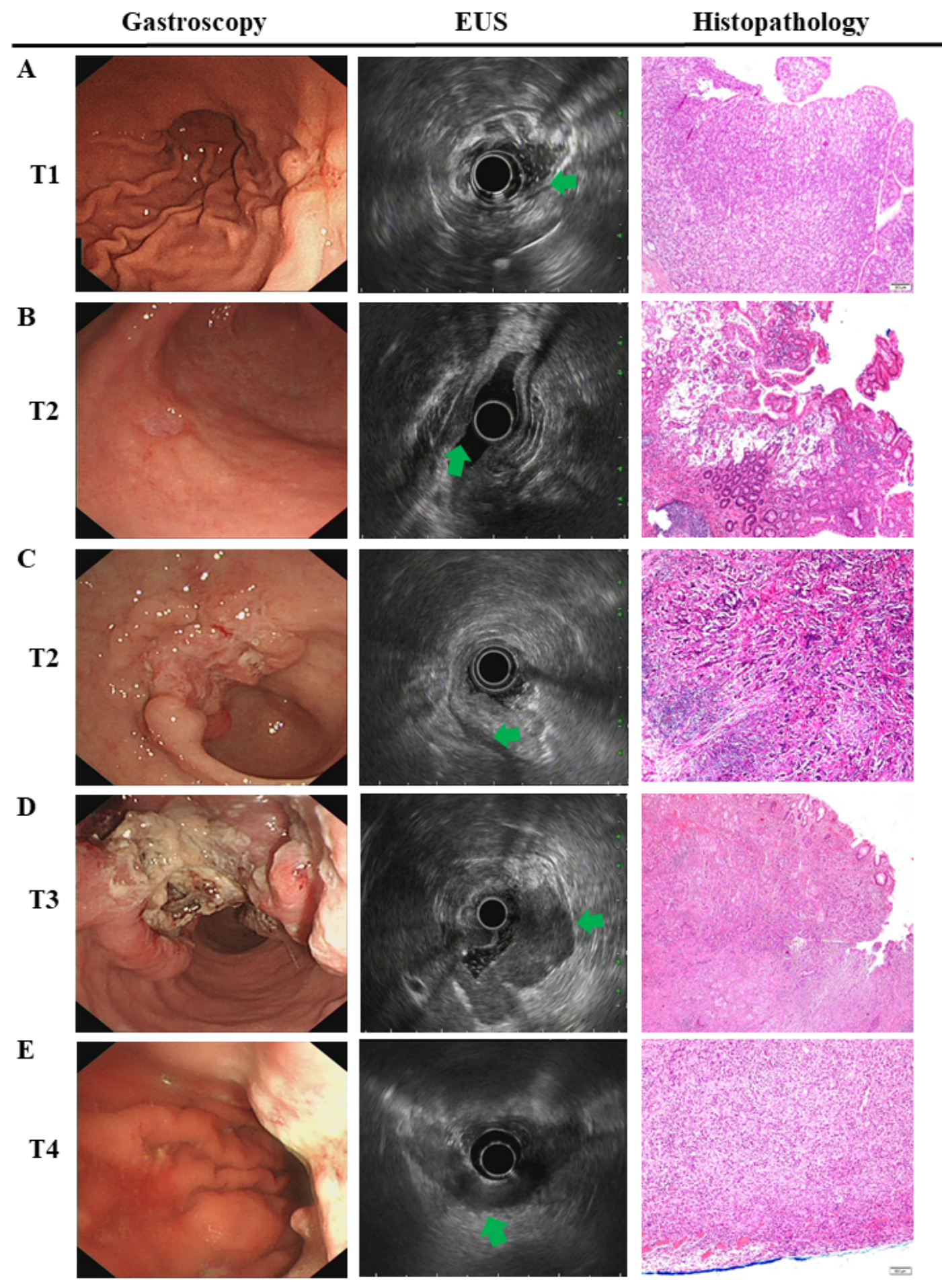

Figure 1

The tumor T stage depend on AJCC 7th/8th edition system and EUS image features for each tumor T stage. (A). Endoscopic image of the lesion showed an ulcer located in the posterior wall of the body with peripheral mucosal consolidation. EUS image showed disappearance of first hyperechoic layer, mild thickness and hypoechoic change of the second hypoechoic layer, and normal third hyperechoic layer (arrows). Surgical resection confirmed poorly-differentiated and partial signet-ring cell gastric cancer 
confined to submucosal layer; (B). Gastroscopy showed an ulcer located in the anterior wall of gastric angle. EUS image of the lesion showed disappearance of the first three layers and companied by muscularis propria visible indistinctly (arrows). The surgical specimen confirmed poorly-differentiated gastric cancer confined to the submucosal layer; (C). Endoscopic image showed a neoplasm located in the anterior wall of the antrum. EUS image of the lesion showed disappearance of the first three layers and companied by muscularis propria obvious thickening (arrows). The surgical specimen confirmed tumor infiltrated to the muscularis propria layer; (D). Endoscopic image of the lesion showed a neoplasm located in the lesser curvature side of the antrum with dirty surface. EUS image showed a thick hypoechoic lesion spreading from the mucosal to muscularis propria layer with an intact serosa layer (arrows). The surgical specimen confirmed tumor infiltrated to the subserosa; (E).Endoscopic image showed a large ulcer located in the upper posterior wall of the gastric body. EUS image showed an obviously thick hypoechoic lesion that spread throughout the entire wall and invaded the serosa infiltration (arrows). The serosal layer was irregularities in the outer edge of the gastric wall. The surgical specimen confirmed lesion confined to the serosal layer.

\section{Supplementary Files}

This is a list of supplementary files associated with this preprint. Click to download.

- Table1.docx

- Table4.docx

- Table3.docx

- Table5.docx

- Table2.docx 